\title{
Bipolar charge transport in organic field-effect transistors: Enabling high mobilities and transport of photo-generated charge carriers by a molecular passivation layer
}

\author{
Andreas Opitz ${ }^{\mathrm{a}, \mathrm{b}, *}$, Matthias Horlet $^{\mathrm{a}}$, Marc Kiwull ${ }^{\mathrm{a}}$, Julia Wagner ${ }^{\mathrm{a}}$, \\ Michael Kraus ${ }^{\mathrm{a}}$, Wolfgang Brütting ${ }^{\mathrm{a}}$ \\ ${ }^{a}$ Institute of Physics, University of Augsburg, Augsburg, Germany. \\ ${ }^{b}$ Institute of Physics, Humboldt University of Berlin, Berlin, Germany.
}

\begin{abstract}
High mobility bipolar charge carrier transport in organic field-effect transistors (OFETs) can be enabled by a molecular passivation layer and selective electrode materials. Using tetratetracontane as passivation layer bipolar transport was realised in the organic semiconductors copper-phthalocyanine, diindenoperylene, pentacene, TIPS-pentacene and sexithiophene and mobilities of up to $0.1 \mathrm{~cm}^{2} / \mathrm{Vs}$ were achieved for both electrons and holes. Furthermore, the trap and injection behaviour was analysed leading to a more general understanding of the transport levels of the used molecular semiconductors and their limitations for electron and hole transport in OFETs. With this knowledge the transistor operation can be further improved by applying two different electrode materials and a light-emitting transistor was demonstrated.
\end{abstract}

Additionally, the effect of illumination on organic field-effect transistors

\footnotetext{
${ }^{*}$ Corresponding author.

Email address: Andreas.Opitz@hu-berlin.de (Andreas Opitz)
} 
was investigated for unipolar and bipolar devices. We find that the behaviour of photo-excited electrons and holes depends on the interface between the insulator and the semiconductor and the choice of contact materials. Whereas filling of electron traps by photo-generated charges and the related accumulation field are the reason for changes in charge carrier transport upon illumination without passivation layer, both types of charge carriers can be transported also in unipolar OFETs, if a passivation layer is present.

Keywords:

molecular semiconductors, unipolar and bipolar transport, interface traps, electrode modification, light emission, light absorption

\section{Introduction}

Molecular or polymeric materials used as organic semiconductor in thinfilm devices have traditionally been reported as either electron or hole transporting materials $[1,2]$. Most of the polymeric materials, like poly-phenylenevinylenes or poly-thiophenes, and some classes of molecular materials, like acenes or phthalocyanines, are typical $p$-conducting materials, whereas fullerenes or fluorinated molecules are mainly $n$-conductive. By contrast, bipolar transport has been reported for high-purity single-crystals as measured by the time-of-flight (TOF) method [3]. In this technique electron-hole pairs are generated by light absorption close to an electrode and one of both carrier types, depending on the sign of the applied voltage, is transported through the whole crystal towards the counter electrode, where its arrival is monitored as transit time. The charge carriers in thin-film devices, however, are generated either by the field effect or by injection. Here the misalign- 
ment of the electrode work function to the respective transport levels can cause substantial injection barriers [4]. Furthermore, the transport in fieldeffect transistors is limited by trap states at the semiconductor/insulator interface. Especially the often used gate oxides, like silica or alumina, have electron traps at their surfaces in the form of hydroxyl groups [5]. By suppressing these interface traps and adjusting the injection barriers for both charge carrier types, injection and transport of electrons and holes are possible $[5,6]$. Thus bipolar transport is also observable in thin-film devices and hence not limited to single-crystals. Beside accumulation and injection the charge carriers in organic field-effect transistors (OFET) can be generated by photo excitation. As mentioned before for the TOF technique here both charge carrier types are generated inside the channel and can account for the transport.

In the following we will show that by applying an aliphatic interlayer bipolar transport in OFETs is possible with a variety of materials, including copper-phthalocyanine $(\mathrm{CuPc})$, diindenoperylene (DIP), pentacene (Pen), 6,13-bis(triisopropylsilylethynyl)-pentacene (TIPS-Pen), and $\alpha$-sexithiophene (6T), that have previously been described as $p$-type molecular semiconductors . Furthermore, we will discuss the influence of electrode materials for electron and hole injection, before considering $n$-type molecular semiconductors. From these observations and the comparison to the transport on bare oxides, limitations for electron and hole transport will be deduced. An improvement of ambipolar transport is achieved by additionally using different source and drain materials for simultaneous electron and hole injection. In the case of the molecular semiconductor DIP light emission from ambipolar 
OFETs was observed. Finally, the effect of illumination on charge carrier transport will be compared. Basic differences appear for unipolar transistors with and without passivation layer using DIP as active material which will be discussed.

\section{Experimental}

Organic field-effect transistors (OFET, for cross-sectional structure see figure 1a) were prepared on highly doped silicon wafers with $320 \mathrm{~nm}$ thermally grown oxide. Additionally a layer of the insulating long chain alkane tetratetracontane (TTC, $\mathrm{C}_{44} \mathrm{H}_{90}$, purchased from Sigma Aldrich, used as received, chemical structure shown in figure 1d) was deposited by thermal evaporation [7] (thickness about $10 \mathrm{~nm}$ ) to separate the charge carrier transport in the molecular semiconductor from the silicon oxide surface. The layers were smoothed by thermal annealing at $60^{\circ} \mathrm{C}$ for 2 hours as shown earlier [8]. Unfortunately TTC shows a good solubility in most organic solvents. So spin coating of an organic semiconductor layer like poly(3-hexylthiophene) or 6,13-bis(triisopropylsilylethynyl)-pentacene (TIPS-Pen) on top of the TTC film dissolves the TTC molecules and removes the passivation layer. Up to now, TTC can be used as passivation layer only for evaporated semiconductors.

The semiconducting material were deposited with a film thickness of $25 \mathrm{~nm}$ as active layer on top of a TTC layer by thermal evaporation. The molecular semiconductors copper-phthalocyanine $(\mathrm{CuPc}), \alpha$-sexithiophene (6T), perfluorinated $\mathrm{CuPc}\left(\mathrm{F}_{16} \mathrm{CuPc}\right.$, all purchased from Sigma Aldrich), diindenoperylene (DIP, from S. Hirschmann, University of Stuttgart), and 
Buckminster fullerene $\mathrm{C}_{60}$ (purchased from Creaphys) were purified by temperature gradient sublimation at least once; in contrast pentacene (Pen) and TIPS-Pen were used as received from Sigma Aldrich. The molecular structures are displayed in figure $1 \mathrm{~b}+\mathrm{c}$. Various electrode materials (aluminium, silver, gold, tetracyanoquinodimethane-tetrathiafulvalene - TTFTCNQ) have been used to analyse transistors in top contact geometry. The metal layers had a thickness of $50 \mathrm{~nm}$ and the organic metal TTF-TCNQ a thickness of $150 \mathrm{~nm}$. Furthermore FETs with two different electrode materials were prepared by a parallactic shadow mask displacement technique [9] (see figure 6a). Thereby the Al layer was evaporated at first and afterwards, with a slight lateral shift, the TTF-TCNQ layer [10]. The whole processing was carried out under high vacuum or under inert atmosphere. Electrical characterization was also performed under high vacuum conditions. The measured transfer characteristics have been analysed by the transfer length method using various channel lengths between 50 and $150 \mu \mathrm{m}[7,11]$.

The surface morphology of evaporated films was determined by scanning force microscopy (SFM) in non-contact mode. Strong differences are observed for the growth behaviour of the molecular semiconductors on top of the TTC passivation layer. Exemplarily the surface morphology is shown in figure 2 for a TTC film as well as for a CuPc film and a DIP film deposited on top of the TTC layer. TTC forms a well crystallized film with a monolayer thickness of about $6 \mathrm{~nm}$, which is close to the length of the molecules [7]. While $\mathrm{CuPc}$ grows only on top of the TTC islands, the DIP grains are exceeding the individual TTC islands $[8,10]$. Additionally, preferred orientations of the $\mathrm{CuPc}$ grains can be seen. They are aligned perpendicular to each other. The 
growth of CuPc on TTC can be considered as some kind of "pseudo-epitaxial" growth [7].

\section{High mobility ambipolar transport}

Measured transfer characteristics of OFETs with CuPc, DIP, Pen, TIPSPen, and 6T as normally " $p$-type" molecular semiconductors are presented in figure 3. For all these semiconductors electron and hole transport, i.e. bipolar transport, is observed. Typical electrode materials for achieving bipolar transport are silver or gold for $\mathrm{CuPc}$ and DIP, silver or aluminium for Pen and TIPS-Pen as well as silver for 6T. If low-work function metals, like Ca or in some cases $\mathrm{Al}$, are used, even unipolar $n$-channel transistors can be fabricated with $\mathrm{CuPc}$ and DIP, while the use of the organic metal TTF-TCNQ allows for achieving unipolar $p$-channel FETs. These results show that TTFTCNQ must have a different interface behaviour than ordinary metals. Even if the reported work function of TTF-TCNQ [12] is comparable to the values of $\mathrm{Au}$ [6], TTF-TCNQ only allows for hole injection, whereas Au allows for both electron and hole injection. The reason for this behaviour might be related to energy level alignment of the here used top contacts together with diffusion of the evaporated metal atoms and associated damages of the organic material [7]. By contrast, only $n$-channel transistors could be achieved for the typical "n-type" materials $\mathrm{C}_{60}$ and $\mathrm{F}_{16} \mathrm{CuPc}$, even with TTF-TCNQ as electrode, as shown by transfer characteristics in figure 4. As summarized in table 1 the determined mobilities for unipolar and ambipolar transport are largely independent of the chosen contact material $[6,7,10]$.

These data clearly show that applying TTC as passivation layer allows for 
achieving high mobility bipolar charge transport in a wide variety of vacuum deposited semiconductor films. Mobilities of up to $0.1 \mathrm{~cm}^{2} / \mathrm{Vs}$ are observed for electrons in TIPS-Pen and DIP as well as for holes in Pen. In comparison to other organic passivation layers, like poly(methyl methacrylate) (PMMA), TTC has the advantage of a lower dielectric constant that reduces polarisation effects in the insulator and a higher surface energy that leads to a more crystalline film growth [7]. The observed asymmetry between electron and hole transport can likely be related to differences in the $\pi-\pi$ overlap between the lowest unoccupied molecular orbital (LUMO) and the highest occupied molecular orbital (HOMO), respectively, of neighbouring molecules, within the crystallites or at the grain boundaries as well as to differences in trap densities, as for example determined for $\mathrm{CuPc}$ and DIP $[8,10]$.

The presented results allow for comparison with single-crystal transport data from literature, which are included in Table 1. Even though ambipolar transport has been reported for CuPc single-crystal FETs [13], the measured hole mobilities were due to device non-idealities of extrinsic origin significantly lower than other values reported for unipolar single-crystal devices [14] and electron mobilities were even lower than in the thin-film devices presented here. In our DIP thin-film transistors the determined mobilities are one order of magnitude higher than the mobilities in DIP single-crystals [15]. In these single-crystals micro cracks represent the major problem. Annealing of Pen single-crystals leads to balanced mobilities in the same order of magnitude as the hole mobility presented here [16]. The low electron mobility in our sample may be related to lower purity of Pen in comparison to $\mathrm{CuPc}$ and DIP. It has to be mentioned that the field-effect mobilities in these 
single crystal devices were determined by the single curve analysis without any correction for contact resistances. For TIPS-Pen and $6 \mathrm{~T}$ no data about ambipolar transport of single-crystals were found in the literature.

To observe bipolar transport in $\mathrm{F}_{16} \mathrm{CuPc}$ and $\mathrm{C}_{60}$ the work function of the electrode would have to be increased further to also allow for hole injection [17]. The observed absence of hole transport is related to a large hole injection barrier into the low lying HOMO level of both materials.

As an overview the energy levels of the different semiconductors used here are summarized in figure 5. If the HOMO energy is higher than $(6.1 \pm$ $0.2) \mathrm{eV}$ no hole injection occurs, as illustrated by the cartoon. Of course, the transition between injecting and blocking contact is gradual. However, for understanding this behaviour in detail more experiments on the nature of the metal top contacts are necessary. To inject holes also in the materials with high ionization potential contact materials with extremely high work function would be necessary, as suggested for organic metals containing other charge transfer molecules than TTF and TCNQ [12].

From the behaviour of the different semiconductors in OFETs with and without a TTC passivation layer an estimate for the electron trap level at bare oxide surfaces can be deduced. Therefore the energy levels for the LUMO are shown in figure 5. If the energy of an additional state is located within the gap of a semiconductor, this state will act as a trap (as illustrated by the cartoon), like it is observed for electrons at bare oxide surfaces. The density of these trap states seems to be so high that electron accumulation exceeding $6 \times 10^{12}$ charges per square centimetre (number of accumulated 
electrons in the here used geometry with an applied effective gate voltage of $+100 \mathrm{~V}$ ) is still not sufficient to fill all traps and to enable electron transport. This happens for all materials up to a LUMO energy of $(3.55 \pm 0.3) \mathrm{eV}$ below the vacuum level. However, for deeper lying LUMO levels, like in $\mathrm{C}_{60}$ and $\mathrm{F}_{16} \mathrm{CuPc}$, this state will not any more act as trap.

The variation of the insulator-semiconductor interaction has been reported before in the literature also for molecular materials and one bipolar example [18]. There the application of polystyrene (PS) as insulating interlayer gave only hole transport for pentacene in contrast to later reports showing bipolar transport in pentacene with PS as passivation layer [19]. This shows that the transport properties are strongly dependent on the materials and the preparation conditions. Here we suggest TTC as general passivation layer for evaporated molecules because TTC was reported to work perfectly also by other groups $[20,21]$. Another approach in the literature was the screening of a large variety of acene derivatives [22]. The transport behaviour in OFETs without passivation layer was described to vary form hole-only via ambipolar to electron-only for changing energy levels. The observed differences of the limiting values of the molecular energy levels for hole- and electron transport (6.1 and $3.55 \mathrm{eV}$ here vs. 5.6 and $3.15 \mathrm{ev}$ in the reference [22]) are related to different electrode materials (TTF-TCNQ here vs. gold in the reference), experimental conditions (mainly work without passivation layer in the reference), and energy level determination (here UPS and IPES data vs. cyclic voltammetry in the 
reference).

To further improve the ambipolar transport behaviour two different electrodes were applied to decrease the injection barrier for electrons and holes separately [10]. The schematic structure of such an OFET with asymmetric ("bilayer") electrodes is illustrated in figure 6a. Here Al and TTF-TCNQ were used for enhanced electron and hole injection, respectively, in comparison to the before mentioned silver electrode. Together with the lower injection barrier a reduction of the threshold voltages for both electrons and holes [10] in the analysed ambipolar DIP OFET results in a significant current increase by four orders of magnitude in the minimum of the transfer characteristic, as shown in figure $6 \mathrm{~b}$.

With these higher currents light emission could easily be detected as shown in figure $6 \mathrm{c}$. By varying the gate voltage at a constant drain voltage the spatial position of the recombination and emission zone can be shifted [23]. Due to the upright standing DIP molecules and the transition dipole moment lying parallel to the long axis of the molecules, the emission is mainly in the substrate plane $[24,25]$. However, this is suitable as a pumping device for organic lasers [26]. As shown in the literature, a further increase of emission efficiency would be possible by separated electron and hole transport as well as an additional emission layer [27].

\section{Transport of light-induced charge carriers}

Beside accumulation and injection, charge carriers can also be generated by dissociation of excitons formed upon light absorption. Therefore we also analysed the illumination effect on unipolar and ambipolar OFETs using the 
molecular semiconductor DIP. As the optical absorption of DIP is located below a wavelength of $580 \mathrm{~nm}$, a blue light-emitting diode (LED) was used for illumination of the OFETs giving an intensity of $4.9 \mathrm{~mW} / \mathrm{cm}^{2}$. The extinction coefficient of an evaporated DIP film and the emission spectrum of the used blue LED are shown in figure 7.

Figure 8a shows the transfer characteristics of a unipolar transistor with electron traps (only dichlorodimethylsilane treatment, no TTC passivation layer) and hole-only injecting electrodes (TTF-TCNQ contacts). Illumination results mainly in a shift of the switch-on (and thus also the threshold) voltage as widely observed in hole-transporting devices [28, 29] and in the occurrence of a hysteresis. The transport of photo-generated electrons and holes is realised in the ambipolar transistor by introducing a TTC layer as shown by the transfer characteristics in figure $8 \mathrm{~b}$. Here a combination of $\mathrm{Al}$ and TTF-TCNQ is used as electrode [10]. Both charge carrier types, either injected or photo-generated, can be transported in this device architecture. The change in the switch-on voltage and the current upon illumination is marginal. Thus the number of photo-generated charge carriers is small in comparison to the high density of accumulated charge carriers in the fieldeffect device.

Unipolar transistors can be obtained in devices with a passivation layer by choosing TTF-TCNQ as electrode material for hole-only injection and with aluminium as electrode for electron-only injection as shown in figure $8 c+d$. A small hole current can also be observed for $\mathrm{Al}$ electrodes, which is related to unintentional changes (like degradation) of this device. In pristine devices only electron injection occurs as shown before [10]. Like in the ambipolar 
transistor the effect of illumination for the injected majority charge carriers is minor. A drastic effect, however, is visible for the charge carrier type whose injection is blocked by the high injection barrier of the electrode, i.e. for holes by using aluminium and for electrons by TTF-TCNQ electrodes. The difference between the Fermi level of the electrode material and the transport level of the blocked carrier types acts as injection barrier. Nevertheless, charge extraction is possible leading to a current flow by photo-generated charge carriers and a large contrast to the dark current in this transistor regime.

The nature of exciton separation is not fully understood at the moment. The average electric field in transport direction is less than $0.5 \mathrm{MV} / \mathrm{cm}$, which is not enough for field-induced dissociation and charge separation of all excitons [30]. However, bimolecular processes leading to dissociation, field-enhanced dissociation at defects or grain boundaries, and the inhomogeneous distribution of charges due to the applied gate field can play an important role. Additionally, photo-assisted carrier injection is possible. Due to the nontransparent top contacts the influence of this light induced effect is confined to the direct contact line which is minor in comparison to the whole channel length.

The cartoons in figure 8e-g illustrate the findings for the illumination of OFETs. The shift in switch-on voltage upon illumination for the device without passivation layer is related to the filling of electron traps at the oxide surface with photo-generated electrons (figure 8e). These trapped and fixed electrons act as interface states $N_{\text {If }}$ and cause additional accumulated holes 
in the transistor channel, which results in the observed shift of the switch-on or threshold voltage $\left(V_{\mathrm{T}}\right)$ following the equation [31]

$$
\Delta V_{\mathrm{T}}=\frac{e \cdot N_{\mathrm{If}}}{C_{\mathrm{Ins}}}
$$

where $e$ is the elementary charge and $C_{\text {Ins }}$ the insulator capacitance. In the case shown here, this yields a density of filled electron traps of about $1 \times 10^{12}$ charges per square centimetre. This is lower than the estimate for the trap density mentioned before, because the oxide surface is covered by silane molecules reacting directly with the trapping OH-groups but not fully passivating them. The passivation layer of TTC fully protects the transport channel from electron traps.

For the bipolar device only minor changes are observed (figure $8 b+f$ ), because the number of photo-generated charge carriers is too small in comparison to the injected charge carriers. There are also only minor changes observed for the current of the injected holes (figure 8f) in the unipolar p-channel device with passivation layer. In contrast, the photo-generated minority charge carriers can be transported and extracted under blocking bias as measured in figure $8 \mathrm{c}+\mathrm{d}$ and illustrated in figure $8 \mathrm{~g}$. Thereby, the current change due to illumination is directly related to the number of photogenerated charge carriers.

\section{Summary}

An aliphatic passivation layer of the long-chain alkane molecule TTC allows for high mobility ambipolar transport for a broad range of molecular 
semiconductors. The observed mobilities are widely comparable to results reported in literature for ambipolar transport in single-crystals. Thus transistors with TTC as interlayer give the same ambipolar performance without the need for preparing single-crystals and OFETs out of these. Our findings show that the classical distinction between hole and electron conducting organic semiconductors is mostly related to the suppression of electron transport due to electron traps at oxide surfaces for the former and the impossibility of hole injection into the high lying HOMO level for the latter.

Additionally the influence of illumination was analysed for unipolar and bipolar OFETs. In the unipolar case with passivation of interface traps the photocurrent is based on the non-injected charge carrier type and the measured current is related only to the photo-generated charge carriers. The origin of the photo-generated charge carriers should be bulk generation by exciton dissociation. Effects like photo-injection and detrapping by sub-gap illumination can be ruled out due to the results with blocking contacts and by the spectrum of the used blue LED lying in the absorption region of the molecular semiconductor, respectively. Further experiments are necessary to transfer this study to photo-transistors with highly absorbing organic materials and optically optimised device layout.

\section{Acknowledgement}

This work was supported by the Deutsche Forschungsgemeinschaft (DFG) within priority programme SPP 1355. 


\section{References}

[1] G. Horowitz, Organic field-effect transistors, Adv. Mater. 10 (1998) 365377.

[2] C. Dimitrakopoulos, P. Malenfant, Organic thin film transistors for large area electronics, Adv. Mater. 14 (2002) 99-117.

[3] N. Karl, in Landolt-Boernstein (New Series) by O. Madelung, M. Schulz, and H. Weiss, updated Edition, Springer Berlin, 2000, Ch. Organic Semiconductors, Group III, Vol. 41E.

[4] T. Yasuda, T. Goto, K. Fujita, T. Tsutsui, Ambipolar pentacene fieldeffect transistors with calcium source-drain electrodes, Appl. Phys. Lett. 85 (2004) 2098-2100.

[5] L. L. Chua, J. Zaumseil, J. F. Chang, E. C. W. Ou, P. K. H. Ho, H. Sirringhaus, R. H. Friend, General observation of n-type field-effect behaviour in organic semiconductors, Nature 434 (2005) 194-199.

[6] A. Opitz, M. Kraus, M. Bronner, J. Wagner, W. Brütting, Bipolar transport in organic field-effect transistors: Organic semiconductor blends versus contact modification, New. J. Phys. 10 (2008) 065006.

[7] M. Kraus, S. Richler, A. Opitz, W. Brütting, S. Haas, T. Hasegawa, A. Hinderhofer, F. Schreiber, High-mobility copper-phthalocyanine field-effect transistors with tetratetracontane passivation layer and organic metal contacts, J. Appl. Phys. 107 (2010) 094503. 
[8] M. Kraus, S. Haug, W. Brütting, A. Opitz, Achievement of balanced electron and hole mobility in copper-phthalocyanine field-effect transistors by using a crystalline aliphatic passivation layer, Org. Electron. 12 (2011) 731-735.

[9] R. Schmechel, M. Ahles, H. von Seggern, A pentacene ambipolar transistor: Experiment and theory, J. Appl. Phys. 98 (2005) 084511.

[10] M. Horlet, M. Kraus, W. Brütting, A. Opitz, Diindenoperylene as ambipolar semiconductor: influence of electrode materials and mobility asymmetry in organic field-effect transistors, Appl. Phys. Lett. 98 (2011) 233304 .

[11] S. Luan, G. W. Neudeck, An experimental study of the source/drain parasitic resistance effects in amorphous silicon thin film transistors, J. Appl. Phys. 72 (1992) 766-772.

[12] Y. Takahashi, T. Hasegawa, Y. Abe, Y. Tokura, G. Saito, Organic metal electrodes for controlled p- and n-type carrier injections in organic fieldeffect transistors, Appl. Phys. Lett. 88 (2006) 073504.

[13] R. W. I. de Boer, A. F. Stassen, M. F. Craciun, C. L. Mulder, A. Molinari, S. Rogge, A. F. Morpurgo, Ambipolar Cu- and Fe-phthalocyanine single-crystal field-effect transistors, Appl. Phys. Lett. 86 (2005) 262109.

[14] R. Zeis, T. Siegrist, C. Kloc, Single-crystal field-effect transistors based on copper phthalocyanine, Appl. Phys. Lett. 86 (2005) 022103.

[15] A. K. Tripathi, J. Pflaum, Correlation between ambipolar transport and 
structural phase transition in diindenoperylene single crystals, Appl. Phys. Lett. 89 (2006) 082103.

[16] T. Takenobu, K. Watanabe, Y. Yomogida, H. Shimotani, Y. Iwasa, Effect of postannealing on the performance of pentacene single-crystal ambipolar transistors, Appl. Phys. Lett. 93 (2008) 073301.

[17] T. Nishikawa, S.-I. Kobayashi, T. Nakanowatari, T. Mitani, T. Shimoda, Y. Kubozono, G. Yamamoto, H. Ishii, M. Niwano, Y. Iwasa, Ambipolar operation of fullerene field-effect transistors by semiconductor/metal interface modification, J. Appl. Phys. 97 (2005) 104509.

[18] M.-H. Yoon, C. Kim, A. Facchetti, T. J. Marks, Gate dielectric chemical structureorganic field-effect transistor performance correlations for electron, hole, and ambipolar organic semiconductors, J. Am. Chem. Soc. 128 (2006) 12851-12869.

[19] N. Benson, C. Melzer, R. Schmechel, H. v. Seggern, Electronic states at the dielectric/semiconductor interface in organic field effect transistors, phys. stat. sol. (a) 205 (2008) 475-487.

[20] S. Ogawa, Y. Kimura, M. Niwano, H. Ishii, Trap elimination and injection switching at organic field effect transistor by inserting an alkane $\left(\mathrm{C}_{44} \mathrm{H}_{90}\right)$ layer, Appl. Phys. Lett. 90 (2007) 033504.

[21] M. Irimia-Vladu, E. D. Glowacki, P. A. Troshin, G. Schwabegger, L. Leonat, D. K. Susarova, O. Krystal, M. Ullah, Y. Kanbur, M. A. Bodea, V. F. Razumov, H. Sitter, S. Bauer, N. S. Sariciftci, Indigo - 
a natural pigment for high performance ambipolar organic field effect transistors and circuits, Adv. Mater. 24 (2012) 375-380.

[22] M. L. Tang, A. D. Reichardt, P. Wei, Z. Bao, Correlating Carrier Type with Frontier Molecular Orbital Energy Levels in Organic Thin Film Transistors of Functionalized Acene Derivatives, J. Am. Chem. Soc. 131 (2009) 5264-5273.

[23] J. Zaumseil, R. H. Friend, H. Sirringhaus, Spatial control of the recombination zone in an ambipolar light-emitting organic transistor, Nature Mater. 5 (2006) 69-74.

[24] A. C. Dürr, F. Schreiber, M. Münch, N. Karl, B. Krause, V. Kruppa, H. Dosch, High structural order in thin films of the organic semiconductor diindenoperylene, Appl. Phys. Lett. 81 (2002) 2276-2278.

[25] U. Heinemeyer, R. Scholz, L. Gisslén, M. I. Alonso, J. O. Ossó, M. Garriga, A. Hinderhofer, M. Kytka, S. Kowarik, A. Gerlach, F. Schreiber, Exciton-phonon coupling in diindenoperylene thin films, Phys. Rev. B 78 (2008) 085210.

[26] T. Takenobu, S. Z. Bisri, T. Takahashi, M. Yahiro, C. Adachi, Y. Iwasa, High current density in light-emitting transistors of organic single crystals, Phys. Rev. Lett. 100 (2008) 066601.

[27] R. Capelli, S. Toffanin, G. Generali, H. Usta, A. Facchetti, M. Muccini, Organic light-emitting transistors with an efficiency that outperforms the equivalent light-emitting diodes, Nature Mater. 9 (2010) 496-503. 
[28] Y. Guo, G. Yu, Y. Liu, Functional organic field-effect transistors, Adv. Mater. 22 (2010) 4427-4447.

[29] C. S. S. Sangeeth, P. Stadler, S. Schaur, N. S. Sariciftci, R. Menon, Interfaces and traps in pentacene field-effect transistor, J. Appl. Phys. 108 (2010) 113703.

[30] P. Peumans, A. Yakimov, S. R. Forrest, Small molecular weight organic thin-film photodetectors and solar cells, J. Appl. Phys. 93 (2003) 36933723.

[31] S. Scheinert, G. Paasch, Fabrication and analysis of polymer field-effect transistors, phys. stat. sol. (a) 201 (2004) 1263-1301.

[32] O. L. Griffith, J. E. Anthony, A. G. Jones, D. L. Lichtenberger, Electronic properties of pentacene versus triisopropylsilylethynyl-substituted pentacene: Environment-dependent effects of the silyl substituent, J. Am. Chem. Soc. 132 (2010) 580-586.

[33] A. Wilke, T. Mizokuro, R.-P. Blum, J. P. Rabe, N. Koch, Electronic Properties of Cu-Phthalocyanine/Fullerene Planar and Bulk Hetereojunctions on PEDOT:PSS, IEEE J. Sel. Top. Quant. 16 (2010) 17321737.

[34] J. Wagner, M. Gruber, A. Hinderhofer, A. Wilke, B. Bröker, J. Frisch, P. Amsalem, A. Vollmer, A. Opitz, N. Koch, F. Schreiber, W. Brütting, High fill factor and open circuit voltage in organic photovoltaic cells with diindenoperylene as donor material, Adv. Funct. Mater. 20 (2010) 4295-4303. 
[35] S. Krause, Determination of the transport levels in thin films of organic semiconductors, Ph.D. thesis, University of Würzburg, online available at: http://opus.bibliothek.uni-wuerzburg.de/volltexte/2009/4047/ (2009).

[36] R. W. Lof, M. A. Vanveenendaal, B. Koopmans, H. T. Jonkman, G. A. Sawatzky, Band-gap, excitons, and coulomb interaction in solid $\mathrm{C}_{60}$, Phys. Rev. Lett. 68 (1992) 3924-3927.

[37] H. Brinkmann, C. Kelting, S. Makarov, O. Tsaryova, G. Schnurpfeil, D. Wöhrle, D. Schlettwein, Fluorinated phthalocyanines as molecular semiconductor thin films, phys. stat. sol. (a) 205 (2008) 409-420. 

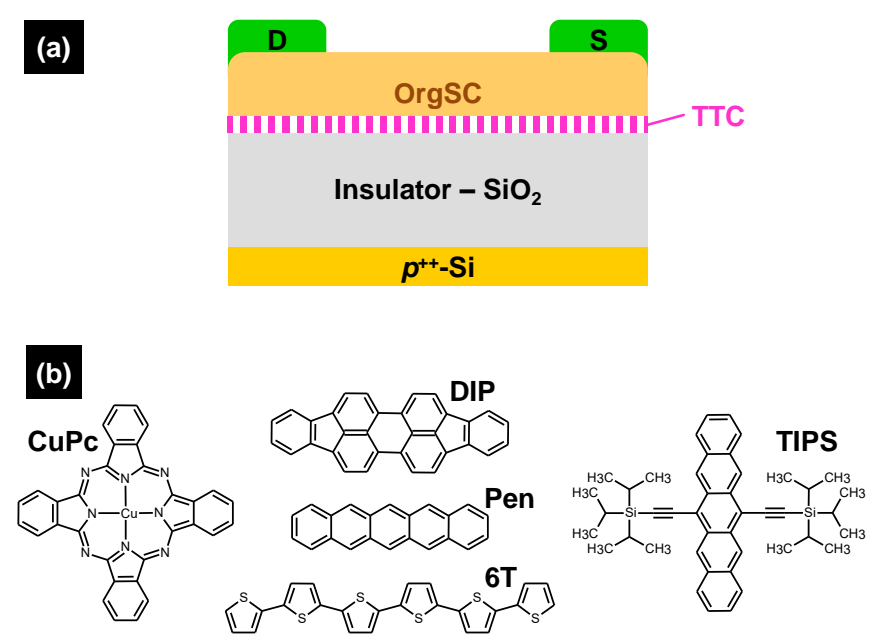

(c)

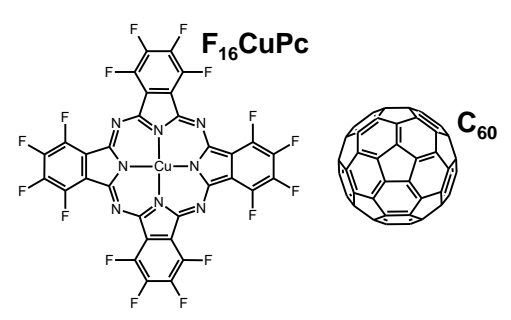

(d) TTC

Figure 1: (a) Cross-sectional structure of the organic field-effect transistor (OFET) in bottom-gate and top-contact geometry. Chemical structure (b) of the typical $p$ type semiconductors copper-phthalocyanine $(\mathrm{CuPc})$, diindenoperylene (DIP), pentacene (Pen), $\alpha$-sexithiophene (6T) and 6,13-bis(triisopropylsilylethynyl)pentacene (TIPS-Pen), (c) of the $n$-type semiconductors perfluorinated CuPc $\left(F_{16} \mathrm{CuPc}\right)$ and Buckminster fullerene $\left(\mathrm{C}_{60}\right)$ as well as $(d)$ of the aliphatic tetratetracontane (TTC) used as passivation layer. 

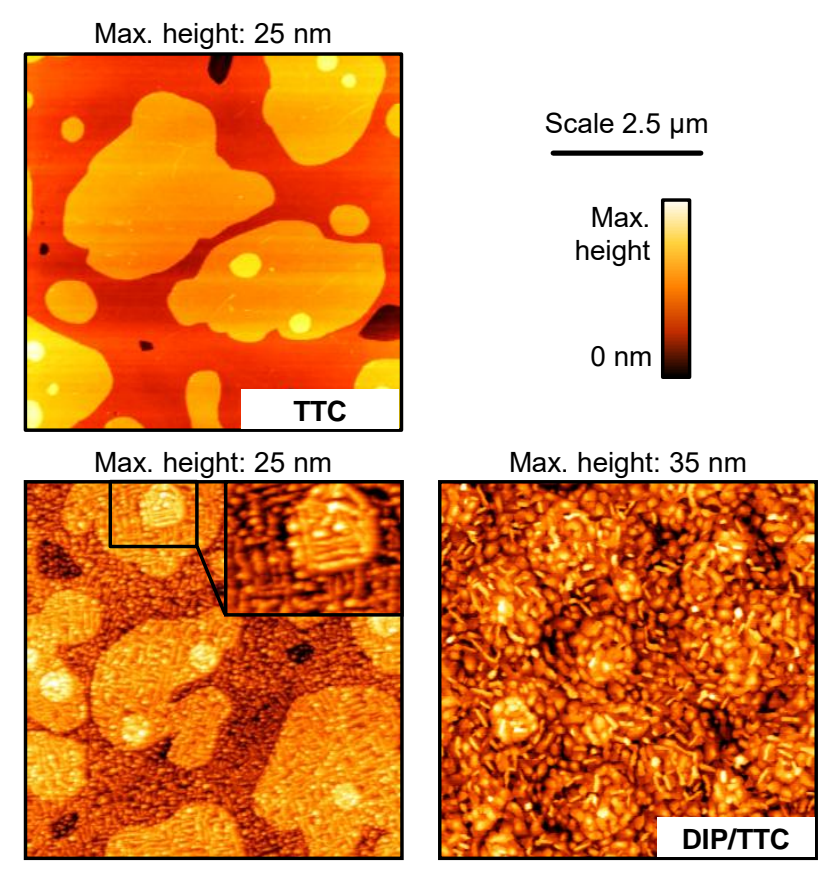

Figure 2: Scanning force microscopy (SFM) images of $10 \mathrm{~nm}$ TTC on oxidised silicon wafer $\left(\mathrm{SiO}_{2} / \mathrm{Si}\right), 25 \mathrm{~nm} \mathrm{CuPc}$ on TTC/SiO $2 / \mathrm{Si}$, and $25 \mathrm{~nm} \mathrm{DIP}$ on TTC/SiO$/ 2 / \mathrm{Si}$. The inset on the image for $\mathrm{CuPc} / \mathrm{TTC} / \mathrm{SiO}_{2}$ is a zoom area to visualize the perpendicular oriented CuPc grains.
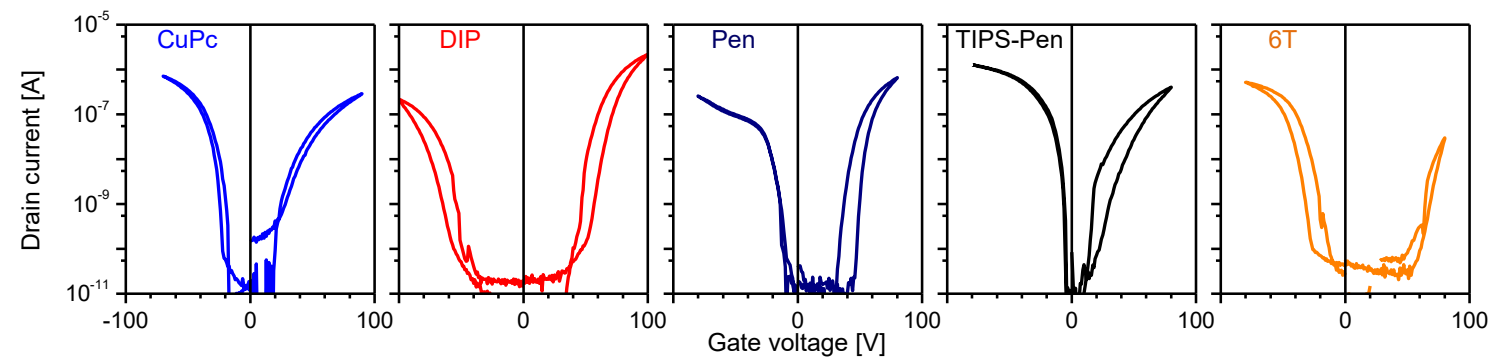

Figure 3: Transfer characteristics of thin-film transistors in the linear region built with the molecular semiconductors copper-phthalocyanine ( $\mathrm{CuPc}, \mathbf{A u}$ contacts), diindenoperylene (DIP, Ag contacts), pentacene (Pen, Al contacts), 6,13-bis(triisopropylsilylethynyl)pentacene (TIPS-Pen, Ag contacts), and $\alpha$-sexithiophene (6T, Ag contacts) using metal contacts for bipolar transport. The drain voltage is $2 \mathrm{~V}$ for $\mathrm{CuPc}$, Pen, TIPS-Pen, and $6 \mathrm{~T}$ OFETs as well as $3 \mathrm{~V}$ for DIP OFETs. The channel length is always $70 \mu \mathrm{m}$. 
Table 1: Summary of bipolar field-effect mobilities determined by transfer length method (TLM) for the evaporated organic semiconductors $\mathrm{CuPc}$, DIP, Pen, TIPS-Pen, 6T, $\mathrm{F}_{16} \mathrm{CuPc}$, and $\mathrm{C}_{60}$ using TTC as interlayer and different electrode materials. Unipolar electron transport was not found with the used electrode materials for Pen and TIPS-Pen. The electron mobility in $6 \mathrm{~T}$ could not be determine by TLM due to the high threshold voltages, as visible in figure 3 . The values in parentheses are extracted from the linear regime of only one transistor using the standard calculation from the slope of a single transfer curve (single curve analysis - SCA). For comparison data for ambipolar transport in single-crystals reported in the literature are added $[13,15,16]$. The method to determine the mobility is also given. TOF stands for time-of-flight measurements.

\begin{tabular}{|c|c|c|c|}
\hline Organic semiconductor & Electrode materials & $\begin{array}{l}\text { Hole mobility } \\
\qquad\left[\mathrm{cm}^{2} / \mathrm{Vs}\right]\end{array}$ & $\begin{array}{l}\text { Electron mobility } \\
{\left[\mathrm{cm}^{2} / \mathrm{Vs}\right]}\end{array}$ \\
\hline $\mathrm{CuPc}$ & $\mathrm{Ag}, \mathrm{Au}$ & $2 \times 10^{-2}$ & $2 \times 10^{-2}$ \\
\hline DIP & $\mathrm{Ag}, \mathrm{Au}$ & $2 \times 10^{-2}$ & $1 \times 10^{-1}$ \\
\hline Pen & $\mathrm{Al}, \mathrm{Ag}$ & $1 \times 10^{-1}$ & $2 \times 10^{-2}$ \\
\hline TIPS-Pen & $\mathrm{Al}, \mathrm{Ag}$ & $4 \times 10^{-2}$ & $1 \times 10^{-1}$ \\
\hline $6 \mathrm{~T}$ & $\mathrm{Ag}$ & $1 \times 10^{-2} \quad\left(1 \times 10^{-2}\right)$ & $\checkmark \quad\left(3 \times 10^{-3}\right)$ \\
\hline $\mathrm{CuPc}$ & TTF-TCNQ & $4 \times 10^{-2}$ & - \\
\hline DIP & TTF-TCNQ & $5 \times 10^{-2}$ & - \\
\hline Pen & TTF-TCNQ & $8 \times 10^{-2}$ & - \\
\hline TIPS-Pen & TTF-TCNQ & $2 \times 10^{-2}$ & - \\
\hline $\mathrm{F}_{16} \mathrm{CuPc}$ & TTF-TCNQ & - & $2 \times 10^{-2}$ \\
\hline $\mathrm{C}_{60}$ & TTF-TCNQ & - & $2 \times 10^{-1}$ \\
\hline Organic semiconductor & Method & Hole mobility & Electron mobility \\
\hline CuPc single-crystal & $\mathrm{SCA}$ & $3 \times 10^{-1}$ & $1 \times 10^{-3}$ \\
\hline DIP single-crystal & TOF & $3 \times 10^{-3}$ & $2 \times 10^{-2}$ \\
\hline Pen single-crystal & SCA & $3 \times 10^{-1}$ & $4 \times 10^{-1}$ \\
\hline
\end{tabular}




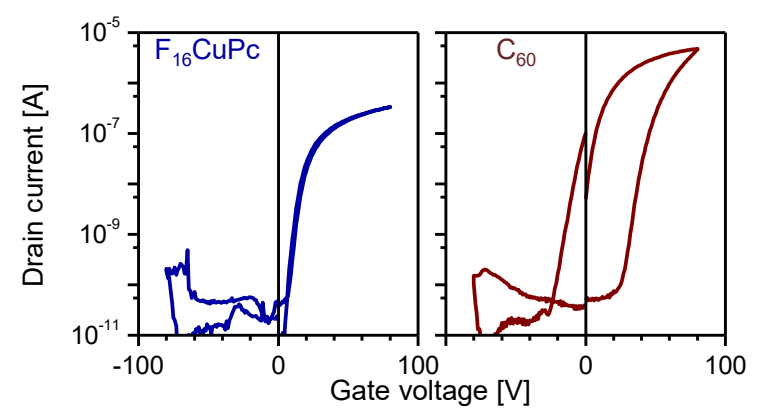

Figure 4: Transfer characteristics of thin-film transistors in the linear region built with the molecular semiconductors perfluorinated copper phthalocyanine $\left(\mathrm{F}_{16} \mathrm{CuPc}\right)$ and Buckminster fullerene $\left(\mathrm{C}_{60}\right)$ using TTF-TCNQ electrodes. The drain voltage is $2 \mathrm{~V}$ for $\mathrm{F}_{16} \mathrm{CuPc}$ OFETs as well as $3 \mathrm{~V}$ for $\mathrm{C}_{60}$ OFETs. The channel length is always $70 \mu \mathrm{m}$.

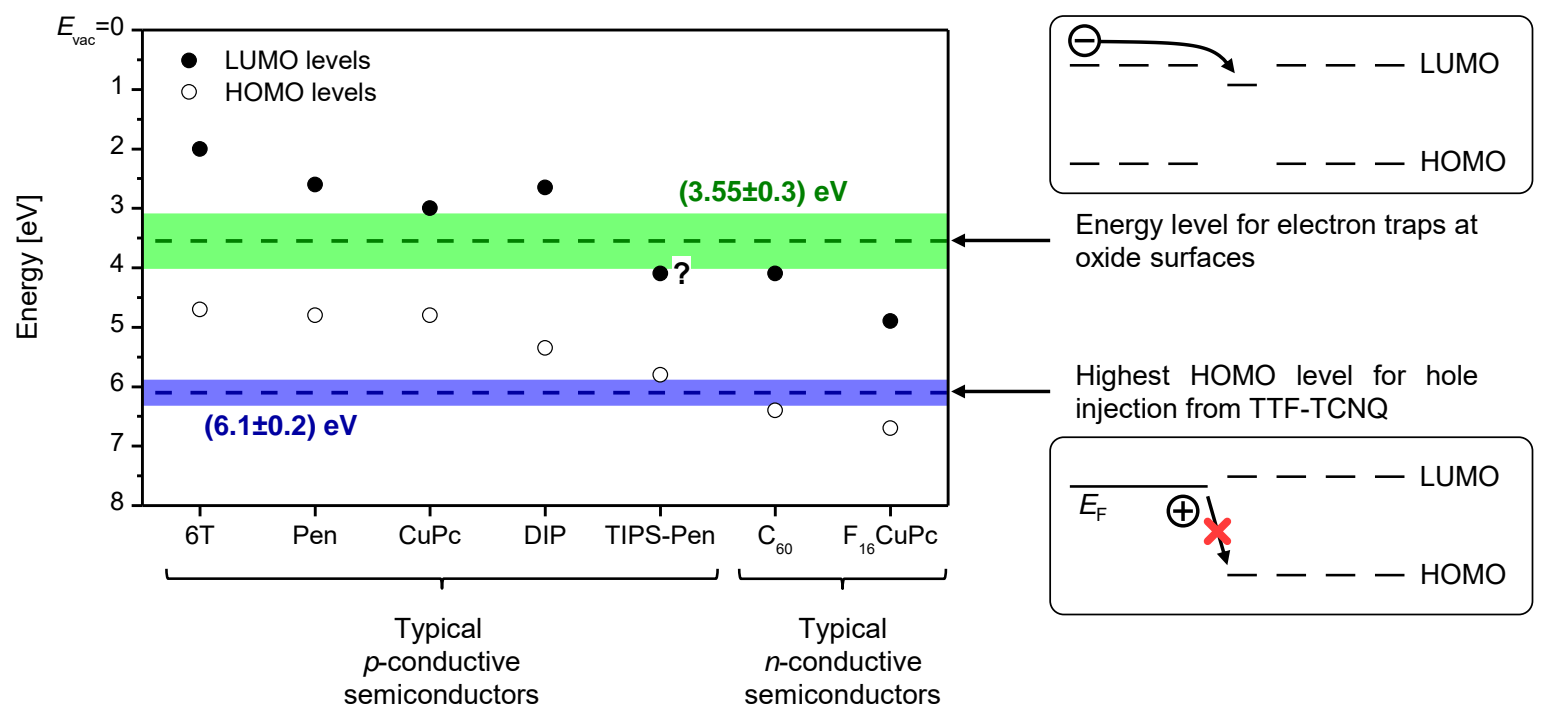

Figure 5: Energy levels of the lowest unoccupied molecular orbital (LUMO) and highest occupied molecular orbital (HOMO) for the used molecular semiconductors [32, 33, 34, $35,36,37]$. Additionally the estimated energy levels for the electron traps due to hydroxyl groups at oxide surfaces and the highest HOMO level where hole injection can occur from TTF-TCNQ are shown with an error region illustrated by the coloured bars. We note that for evaporated TIPS-Pen no data were found in the literature. The preparation of TIPS-Pen films from solution or under ambient conditions possibly suppresses electron transport in this material. On the other hand the LUMO level might be inaccurate as unoccupied states are not directly accessible by most techniques. On the right-hand side the process of electron trapping to hinder the electron transport and the suppression of hole injection are illustrated by cartoons. 
(a)

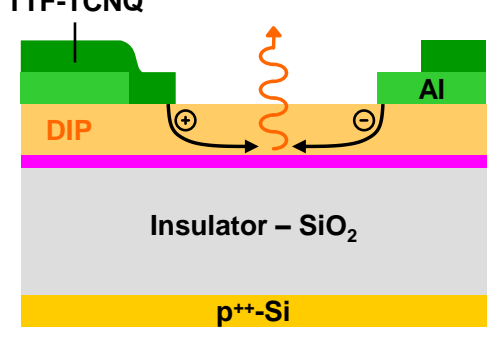

(c)

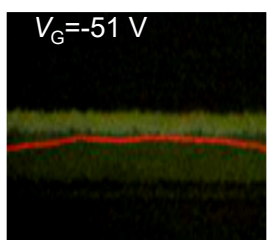

(b)

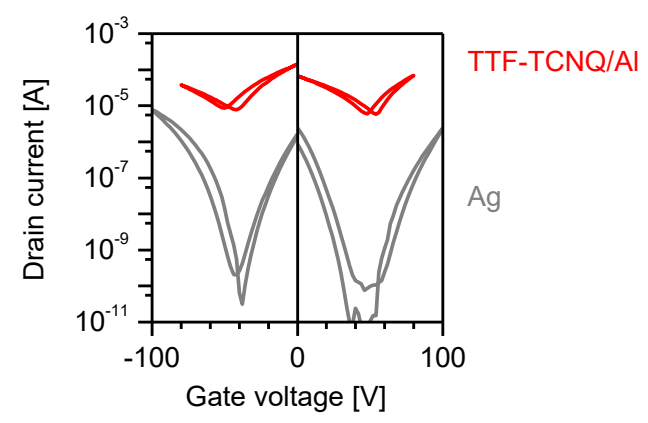

Figure 6: (a) Structure of an ambipolar OFET containing two different electrode materials. Additionally electron and hole paths as well as the emission of light from the recombination zone are illustrated. (b) Comparison of the ambipolar transfer characteristic of a DIP OFET in the saturation regime for two different electrode configurations, silver electrodes and "bilayer" electrodes of $\mathrm{Al}$ and TTF-TCNQ (drain voltage $\pm 100 \mathrm{~V}$, channel length for Ag electrodes $70 \mu \mathrm{m}$, for "bilayer" electrodes $63 \mu \mathrm{m}$ ). (c) Light emission from DIP OFET with "bilayer" electrodes for different gate voltages at a drain voltage of $-100 \mathrm{~V}$ to show the capability to move the emission zone through the channel. 


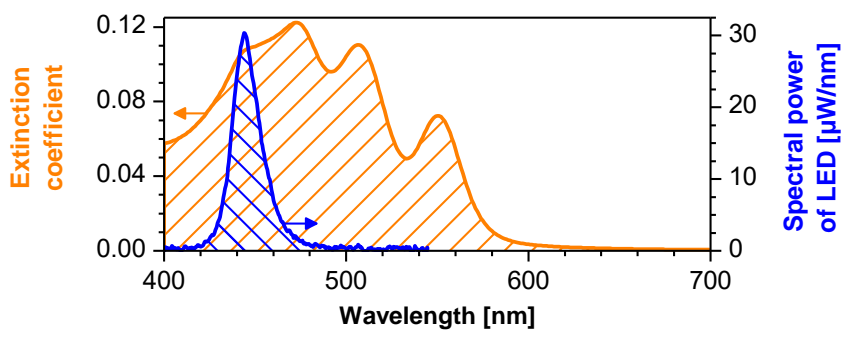

Figure 7: Extinction coefficient of the molecular semiconductor diindenoperylene in evaporated thin films and spectral power of blue light-emitting diode used for illumination of OFETs.
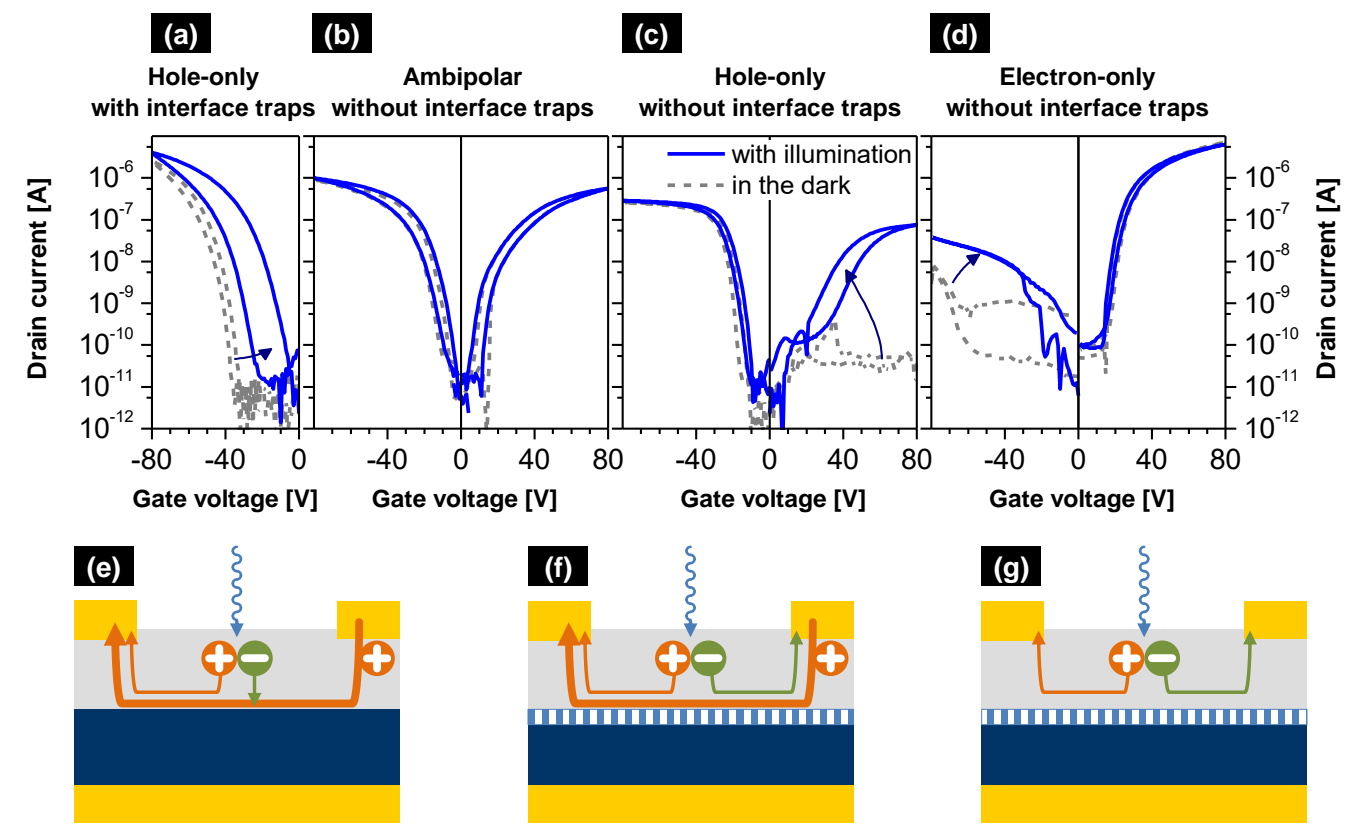

Figure 8: Transfer characteristics for different OFET layouts with and without illumination: (a) hole-only transistor without passivation layer and with hole-only injecting electrodes, (b) ambipolar transistor with passivation layer and electrodes to inject electrons and holes, (c) hole-only transistor with passivation layer and hole-only injecting electrodes, (d) electron-only transistor with passivation layer and electron-only injecting electrodes. Schematic drawing of the illumination effect for hole-only transistors without passivation layer/with interface traps (e) and with passivation layer/without interface traps for injecting (f) and blocking biasing $(\mathrm{g})$. 